\title{
Noise in respiratory signals influences dynamic respiratory system compliance analysis: A simulation study
}

\author{
Zhanqi Zhao ${ }^{1}$, Geoff Chase ${ }^{2}$, Knut Möller ${ }^{1}$ \\ ${ }^{1}$ Department of Biomedical Engineering, Furtwangen University, VS-Schwenningen, Germany \\ ${ }^{2}$ Department of Mechanical Engineering, University of Canterbury, Christchurch, New Zealand \\ zhanqi.zhao@hs-furtwangen.de
}

\begin{abstract}
The aim of the study was to verify the influence of noise in respiratory signals to the analysis of dynamic respiratory system compliance $\left(C_{r s}\right)$. The respiratory signals were simulated under volume control ventilation mode. Volume-dependent $C_{r s}$ was set to an exponential function. White noise and colored noise at signal-to-noise ratio (SNR) of $+\infty, 60 \mathrm{~dB}$ and $30 \mathrm{~dB}$ were added to the simulated respiratory data. Three dynamic methods for $C_{r s}$ analysis (the SLICE method, the dynostatic algorithm, and the adaptive slice method) were examined. We found that the estimates from different dynamic methods were different due to the presence of noise in the respiratory signals. Approaches that increase the SNR may improve the accuracy of the volume-dependent $C_{r s}$ analysis.
\end{abstract}

Keywords: dynamic respiratory system compliance; signal-to-noise ratio; dynamic methods; noise; respiratory signals

\section{Introduction}

The analysis of dynamic respiratory system compliance $\left(\mathrm{C}_{\mathrm{rs}}\right)$ is important for the treatment of patients in intensive care units [1]. Compliance is actually not constant even within one breath but may be depended on volume, flow and respiratory frequency [2]. Volume-dependent compliance may indicate the correctness of ventilator settings and therefore should be monitored at the bedside [3].

There were different methods proposed to calculate volume-dependent $\mathrm{C}_{\mathrm{rs}}$ based on the first order equation of motion [4-6]. In a previous study, we found that the estimates delivered by these dynamic methods are not identical [6]. We hypothesized that noise in the respiratory signals influences the analysis of volume-dependent $C_{r s}$ and causes the difference in estimates of these methods. In the present study, we simulated noise in respiratory data at different signal to noise ratio (SNR) levels in order to prove our hypothesis.

\section{Methods}

\section{A. Breath Simulation}

The detail of the simulated respiratory data was described in a previous study [6]. In brief, a breath under volume controlled ventilation was simulated with MATLAB (The MathWorks Inc., Natick, MA, USA). Tidal volume, inspiration-to-expiration ratio and ventilatory frequency were set to $500 \mathrm{ml}, 1: 2$ and $10 \mathrm{~min}^{-1}$, respectively. Airway resistance and $\mathrm{C}_{\mathrm{rs}}$ were set to $2.22 \times 10^{-3} \quad \mathrm{cmH}_{2} \mathrm{O} \cdot \mathrm{s} \cdot \mathrm{ml}^{-1}$ and $30+$ $20 \cdot e^{- \text {Volume }(\mathrm{t}) / 100} \mathrm{ml} \cdot \mathrm{cmH}_{2} \mathrm{O}^{-1}$ to simulate volume dependent
$\mathrm{C}_{\mathrm{rs}}$. Both white noise and color noise were added to the respiratory data (airway pressure, airway flow and volume) with three different SNR $(+\infty, 60 \mathrm{~dB}, 30 \mathrm{~dB})$. The white noise had a mean value of 0 and a standard deviation of $\sigma$ :

$$
\sigma=A \cdot \sqrt{\frac{\left(10^{-S N R / 10}\right)}{2}}
$$

where $A / \sqrt{2}$ is the effective value of the respiratory signals. The color noise was generated by filtering the white noise with a low pass filter.

\section{B. The analysis of $\mathbf{C}_{\mathrm{rs}}$}

Three dynamic methods to calculate $\mathrm{C}_{\mathrm{rs}}$ were applied in the study, namely the SLICE method [5], the dynostatic algorithm (DSA) [4], and the adaptive slice method (ASM) [6]. These dynamic methods linearize the volume-dependent $C_{r s}$ by dividing the respiratory data into several iso-volume intervals and assuming the $\mathrm{C}_{\mathrm{rs}}$ to be constant within every interval. The SLICE method applies least-squares fit method to each interval [5]. The DSA calculates first the alveolar pressure by subtracting resistive pressure from tracheal pressure. $\mathrm{C}_{\mathrm{rs}}$ is then calculated by dividing volume with alveolar pressure in each volume interval [4]. The ASM analyzes the $\mathrm{C}_{\mathrm{rs}}$ in certain intervals that are selected according to the confidence interval of the fitting parameters [6].

Relative error was defined as the absolute difference between calculated $\mathrm{C}_{\mathrm{rs}}$ and preset $\mathrm{C}_{\mathrm{rs}}$ divided by preset $\mathrm{C}_{\mathrm{rs}}$. Mean relative error was defined as sum relative error divided by the number of estimates delivered with one dynamic method. For example, the SLICE method returned $6 \mathrm{C}_{\mathrm{rs}}$ values at 6 different volume levels, so that the number of estimates was 6 .

\section{Results}

Volume-dependent $\mathrm{C}_{\mathrm{rs}}$ is calculated with SLICE, DSA and ASM methods. The results are plotted against volume in Fig. 1. When no noise is presented in the respiratory data (Fig. 1, top, SNR $=+\infty$ ), the estimations of different dynamic methods are almost identical. When the noise ratio increases to $30 \mathrm{~dB}$ (Fig. 1, middle and bottom), the dynamic methods deliver the estimations with considerable deviation. The observations are similar both in data with white and colored noise.

Mean relative error of each method and total error at each SNR level are summarized in Table 1. The error of the estimates increases when the SNR decreases.

\section{Discussion}

In the present study, noise in the respiratory signals were confirmed to be the main reason influencing the analysis 
of volume-dependent $\mathrm{C}_{\mathrm{rs}}$. The estimates of the dynamic methods were similar at high SNR level. High noise component in the respiratory data diversified the results of the methods (Fig. 1 and Table 1).

The dynamic methods are based on the simple first order equation of motion but showed the potential of clinical applications [7-8]. A close monitoring of volumedependent $\mathrm{C}_{\mathrm{rs}}$ may lead to a new bedside diagnostic tool in the intensive care unit. However, the $\mathrm{C}_{\mathrm{rs}}$ values are not consistent, depending on which method is chosen for analysis. The respiratory signals measured in daily clinical routine consist of real signals and noise (etc. from devices, environment). The presence of noise in the respiratory signals is inevitable but can be reduced through
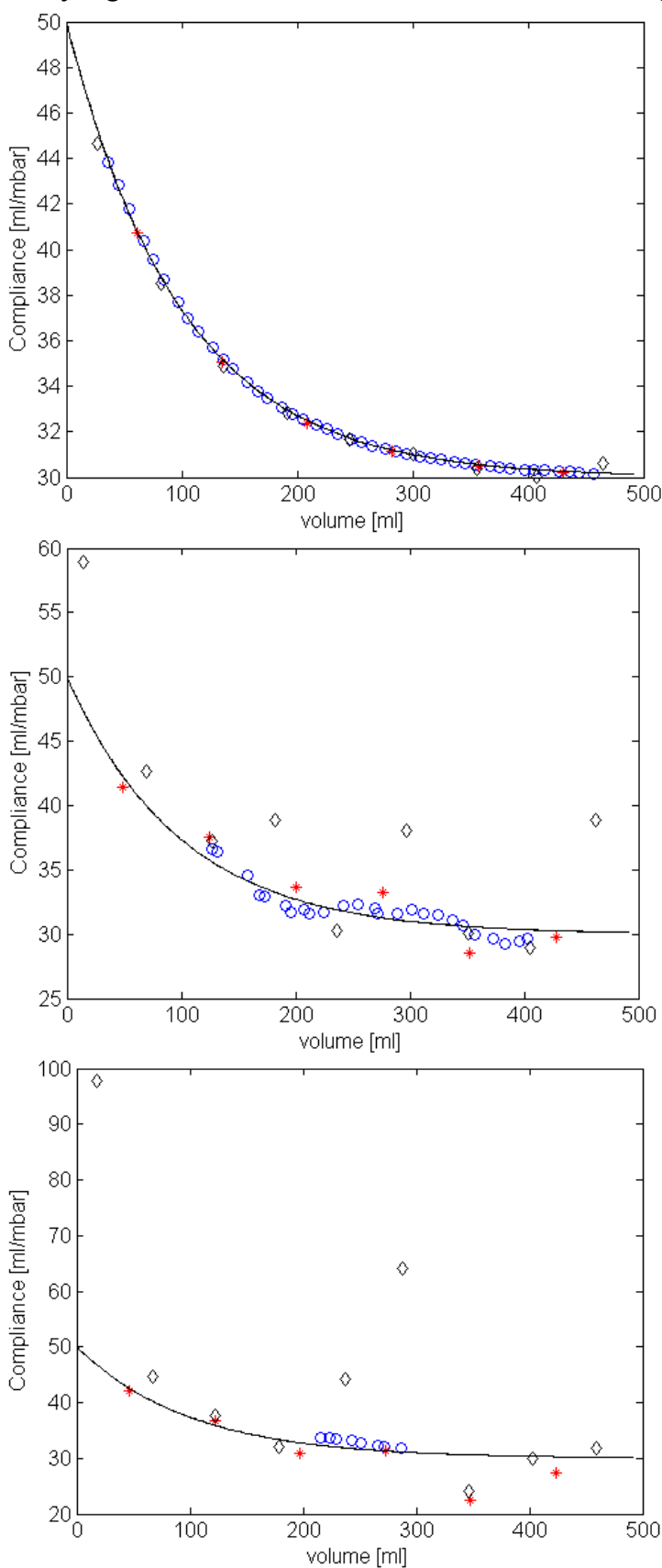

Fig. 1. Calculated volume-dependent $\mathrm{C}_{\mathrm{rs}}$ with SLICE (red $*$ ), DSA (black $\diamond$ ) and ASM (blue $\circ$ ) at a SNR level of $+\infty$ (top) and $30 \mathrm{~dB}$ (white noise, middle and colored noise, bottom). The black line indicates the preset $\mathrm{C}_{\mathrm{rs}}$ value. filtering and some other techniques [9], so that the analysis of $\mathrm{C}_{\mathrm{rs}}$ will become more accurate according to the finding of the present study. In further studies, approaches to raise the SNR in the signals should be examined.

The main limitation of the present study is that the conclusion is based on the simulation. Unfortunately, real $\mathrm{C}_{\mathrm{rs}}$ values are not available in animal experiments or in studies with patients. Further validation studies should be conducted with physical models (phantoms).

Table 1: Mean relative error of different dynamic methods and total error at different SNR levels.

\begin{tabular}{|l|l|l|l|l|}
\hline \multicolumn{2}{|l|}{ Noise $\backslash$ Method } & SLICE & DSA & ASM \\
\hline SNR $=+\infty$ & & 0.002 & 0.007 & 0.001 \\
\hline \multirow{2}{*}{$\begin{array}{l}\text { SNR }=60 \\
\text { dB }\end{array}$} & White & 0.002 & 0.007 & 0.007 \\
\cline { 2 - 5 } & Colored & 0.004 & 0.010 & 0.023 \\
\hline $\begin{array}{l}\text { SNR }=30 \\
\text { dB }\end{array}$ & White & 0.044 & 0.142 & 0.025 \\
\cline { 2 - 5 } & Colored & 0.086 & 0.190 & 0.031 \\
\hline
\end{tabular}

\section{Conclusion}

The estimates from different dynamic methods may be different due to the presence of noise in the respiratory signals. Approaches that increase the SNR may improve the accuracy of the volume-dependent $\mathrm{C}_{\mathrm{rs}}$ analysis.

\section{Acknowledgement}

This work was partially supported by the FP7-IRSES 318943 (eTime).

\section{Bibliography}

[1] Amato, MB. et al.: Effect of a protective-ventilation strategy on mortality in the acute respiratory distress syndrome, $N$ Engl J Med, vol. 338, pp. 347-54, 1998.

[2] D'Angelo, E. et al.,: Respiratory mechanics in anesthetized paralyzed humans: effects of flow, volume, and time, J Appl Physiol, vol. 67, pp. 2556-64, 1989.

[3] Mols, G. et al.: Volume-dependent compliance in ARDS: proposal of a new diagnostic concept, Intensive Care Med, vol. 25, pp. 1084-91, 1999.

[4] Karason, S., et al.: A new method for non-invasive, manoeuvre-free determination of static pressure-volume curves during dynamic/therapeutic mechanical ventilation, Acta Anaesthesiol Scand, vol. 44, pp. 578-85, 2000.

[5] Guttmann, J. et al.: Determination of volume-dependent respiratory system mechanics in mechanically ventilated patients using the new SLICE method, Technol Health Care, vol. 2, pp. 175-191, 1994.

[6] Zhao, Z. et al.: Adaptive SLICE method: an enhanced method to determine nonlinear dynamic respiratory system mechanics, Physiol Meas, vol. 33, pp. 51-64, 2012

[7] Mols, G. et al.: Different strategies to keep the lung open: a study in isolated perfused rabbit lungs, Crit Care Med, vol. 30, pp. 1598-604, 2002.

[8] Kessler, V. et al.: Analysis of nonlinear volumedependent respiratory system mechanics in pediatric patients, Pediatr Crit Care Med, vol. 1, pp. 111-8, 2000.

[9] Zhao, Z. et al.: Assessment of a volume-dependent dynamic respiratory system compliance in ALI?ARDS by pooling breathing cycles, Physiol Meas. Vol. 33, pp. N61-7, 2012 